\title{
Research on Renewable Resources in Circular Economy based on Intelligent Processing Technology and Product Life Cycle Analysis
}

\author{
Longfei Yu, Shifan Zhu* \\ College of Mechanical and Electrical Engineering, Harbin Engineering University, Harbin 150001, PR \\ China \\ *Corresponding author
}

\begin{abstract}
To achieve more real-time and efficient management of renewable resources and ensure the economic efficiency and environmental friendliness of the process, the renewable resources management mode based on intelligent processing and life cycle analysis was discussed. With the advantages of intelligent processing technology in perception, data collection, information network transmission and intelligent processing, the theory and method of life cycle analysis on environmental impact assessment were applied to the field of renewable resources management, so as to realize efficient and intelligent management of renewable resources. Meanwhile, the environmental effects of various renewable resources should be taken into account, and their respective advantages should be given full play to provide better services for the management of renewable resources. Finally, the combined application of the two was explored by taking the data as the integrating point.
\end{abstract}

Keywords: life cycle analysis; Intelligent processing technology; Renewable resources

\section{Introduction}

The world today is faced with such problems as the rapid increase of the global population, the shortage of resources as well as environmental pollution.Traditional economy, as a one-way linear and open economic process of "resource-product-pollution emission", no longer adapts to the current economic developmentrequirements. Under such circumstances, the concept of circular economy was born at the right moment.Circular economy is a closed-loop feedback cycle process of "resources-products-renewable resources", which advocates an economic development mode in harmony with the environment, emphasizes the reuse of waste renewable resources, and reduces the impact on the environment[1].

The circular economy aims to develop the economy based on material recycling, regeneration and utilization, and follows the principles of Reduce, Ruse and Recycle, namely the 3R principle, whose basic concept is the repetitive utilization of renewable resources.Here, renewable resources refer to various wastes produced in the social production and living consumptionprocess, which have lost all or part of their original use value and can regain their use value after recycling and processing[2].

Renewable resources have their particularities, and their essence is the waste with reuse value after being treated by certain technical means. The nature of the waste determines that the renewable resources must have a direct or indirect impact on the environment in the recycling and treatmentprocess. Therefore, the management of renewable resources is different from that of common resources, because managers need to not only know about the basic attribute information of renewable resources (such as type, name, and origin) but also understandthe state of the whole process from the production, transportation to processing, and determine the impact of the renewable resources on the environment in the recycling process.In this paper, the renewable resources management modebased on intelligent processing and life cycle analysis gave full play to the advantages of the both, and realized the efficient and reliable management of the renewable resources while taking into account their environmental impact. Finally, the combined application of intelligent processing and life cycle analysis in the field of renewable

ISSN: 0010-8189 
resources management was discussed by taking data as the bonding point, in order to achieve real-time, efficient, economic and environmental management of renewable resources, and finally form an intelligent and environmentally friendly renewable resources management mode[3-5].

\section{Intelligent processing}

Intelligent processing is established based on the existing Internet, and its core and foundation are still the Internet, but its user end extends to between items, greatly expanding the Internet.According to the operation process of intelligent processing, it can be divided into perception layer, network layer and application layer[6-8].The perception layer collects all kinds of data and reports the information. The network layer provides data transmission channels and certain data processing functions; The application layer combines intelligent processing with specific industry applications.Intelligent processing takes advantage of all sorts of sensing technologies to collectthe information of the object itself and the surrounding environment and upload the data to the platform in real time, and on the basis of the existing Internet, an ubiquitous networkwhose scope of influencecan touch all the items. At the same time, perfect intelligent processing also should have corresponding intelligent processing ability, and realize the intelligent control of objects as well as real-time and accurate processing of events. The above characteristics of intelligent processing make it have unique advantages in renewable resources management, so as to realize real-time and efficient supervision. The architecture of the intelligent processing technology is shown in Figure 1.

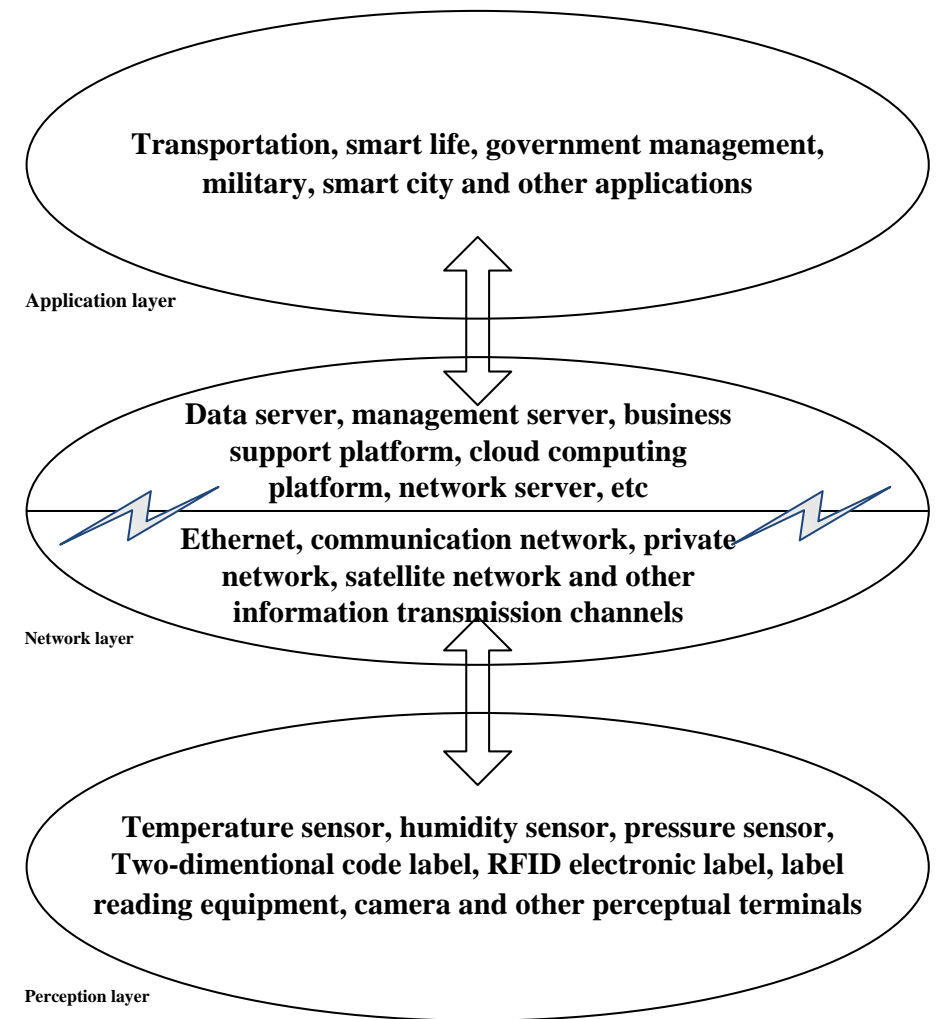

Figure 1 Architecture of intelligent processing technology

\section{Application of intelligent processing in renewable resources management}

The types of renewable resources are complex, involving many aspects of industrial production and daily life. Different types of renewable resources have different characteristics in the generation source, recycling way, transportation mode and processing means.In the recyclingprocess, in addition to the necessary policy and institutional guarantee, it also needs some technical means to effectively manage the whole process. Traditional 
renewable resources management methods mostly depend on fixed recovery sites, where the relevant static data of renewable resources, such as the origin, types, pretreatment methods and other information, are recorded, and the relevant laws and regulations are relied on to provide support and supervision. This way has the following drawbacks: low work efficiency and lack of orderly management;Low recycling rate of renewable resources and serious waste of resources;delayed recovery caused by the lack of information sharing; The management work is carried out by the branches as units, and there is a lack of overall monitoring of the whole recyclingprocess, so the tracking and tracing of renewable resources cannot be realized[9].Therefore, people need more efficient and reliable management methods, so intelligent processing has become the best tool to solve the management of renewable resources with its perception and processing ability of massive data.

The advantage of intelligent processing lies in its ability to detect and control the physical world.It connects all kinds of information sensing devices through the Internet, through which the information interaction between objects is completed, and finally realizes the identification, positioning and monitoring of objects.In the field of renewable resources, intelligent processing mainly uses RFID electronic tags, GPS and other technologies to monitor the real-time status and position of recyclable renewable resources, and then completes the effective management of recycling and utilization of renewable resources. This work is mainly realized from three perspectives.

\subsection{Resource Collection}

Various information collection means of intelligent processing sensing layer are used to collect the necessary information of renewable resources (including type, time, place, state, etc.), and the information is transmitted to the information management platform of renewable resources, through which various information can be viewed and the equipment can be managed.In this process, the information collection means of intelligent processing of the perception layer (including RFID technology, bar code technology, GPS technology, etc.) play a major role.

\subsection{Resource Transportation.}

The renewable resource management at this stage is similar to the application of intelligent processing in the field of logistics. By collecting and reporting the basic attributes of goods in the transportation process and relying on the real-time location information obtained by GPS, the data is monitored by the information management platform and visually displayed and managed in the form of a map

\subsection{Resource processing.}

The processing of renewable resources is the last station of the transportation of renewable resources, where the renewable resources are handled.The RFID reader is installed on the recycling device to read the RFID tag information and automatically classify it. The final processing is unified through the management center. Renewable resources become new products, which provides a necessary basis for the traceability ofrenewable resources.

The service provided by intelligent processing for the field of renewable resources relies on the development level of intelligent processing technology. As far as the current technologies are concerned, renewable resources management based on intelligent processing is mostly realized by means of one-dimensional bar code, two-dimensional bar code as well as RFID electronic tags.Li Xianganalyzed the research status of urban renewable resources and the characteristics of intelligent processing technology, and combined with the current situation and existing problems of renewable resources management in Wuhan, he proposed the use of advanced intelligent processing technology to build an urban renewable resources management information platform in Wuhan.The information platform is composed of a smart collection module, smart transportation module and smart processing module. With the help of RFID tag, GPS and other technologies, the whole process of garbage classification collection, classification transportation and classification processing can be automatically monitored and 
managed.This platform is a typical mode that the intelligent processing technology was applied in the field of renewable resources:The sensing terminal uses sensors, RFID and other technologies to collect data. The transportation process is monitored in real time with GPS and other technologies, and the processing process is managed in real time. All the processes are managed by the background information management center in a unified manner. With the help of cloud computing and other technologies, the massive data from the perception layer is managed, analyzed and processed.

The advanced application of intelligent processing is mainly reflected in the perceptual technical means of the perceptual layer.In the supervision process of renewable resources, in addition to using labels to mark items, various sensors such as temperature sensors, humidity sensors and pressure sensors can also be used to collect the temperature, humidity, pressure and other state information of items and their environment, thus realizing more accurate supervision.

\section{Life cycle assessment}

Life Cycle Assessment is a method of assessing the environmental impact of a product at all stages of its life cycle from birth to death[]. The term "Life cycle" refers to all related activities throughout the life of the product, including production, use and maintenance, as well as final disposal.It also includes the process of acquiring raw materials that is easy to be ignored by people.

Lifecycle assessment can help decision makers select products or processes with the least environmental impact, and life cycle assessment information can be used in combination with other factors such as cost and performance data in the selection process[26]. The goal of life cycle assessment is not simply to achieve "standardized discharge", but to improve the environmental performance of products at the source and make them more environmentally compatible at a deeper level.It can also be said that the idea of life cycle assessment guides a new way of environmental protection, that is, clean production, from the acquisition of raw materials to the production of products, to the final disposal of waste products, every process is in maximum harmony with the environment.Life cycle assessment can help enterprises understand the environmental factors related to their production activities, and actively establish a perfect environmental management system. Therefore, it shouldersthe environmental responsibility andformulates reasonable environmental management policies. Secondly, life cycle assessment can help enterprises find the root of environmental problems related to enterprise activities and products, seek solutions from the source, improve the utilization rate of material and energy, decrease waste emissions, reduce the cost as well as the product potential environmental risks, and then realize the overall control of the whole process.

\section{Application of life cycle assessment in renewable resources management}

Lifecycle assessment can help decision makers select products or processes with the least environmental impact, and lifecycle assessment information can be used in combination with other factors such as cost and performance data in the selection process. The goal of life cycle assessment is not simply to achieve "standardized discharge", but to improve the environmental performance of products at the source and make them more environmentally compatible at a deeper level.It can also be said that the idea of life cycle assessment guides a new way of environmental protection, that is, clean production, from the acquisition of raw materials to the production of products, to the final disposal of waste products, every process is in maximum harmony with the environment.Life cycle assessment can help enterprises understand the environmental factors related to their production activities, and actively establish a perfect environmental management system. Therefore, it shouldersthe environmental responsibility andformulates reasonable environmental management policies. Secondly, life cycle assessment can help enterprises find the root of environmental problems related to enterprise activities and products, seek solutions from the source, improve the utilization rate of material and energy, decrease waste emissions, reduce the cost as well as the product potential environmental risks, and then realize the overall control of the whole process. 


\section{Exploration on the combined application of intelligent processing and life cycle assessment in the} renewable resources management

By analyzing the basic concepts and operation modes of intelligent processing and life cycle assessment, it can be concluded that data is the integrating point of both.Intelligent processing is a technology based on data, and most of its data is collected by relying on its own perception layer. Through data transmission at the network layer, the data is finally managed and analyzed by the information system. However, in the standard technical frame of life cycle assessment, inventory analysis is a step that plays an important fundamental role, because it lists variousinputs such as energy, water and materials as well as various outputs of emissions such as air, solid waste, and waste waterin different stages of the life cycle, and then they are analyzed by follow-up evaluation steps.The lifecycle analysis method is carried out by relying on inventory data, which overlaps with perceptual data acquired by intelligent processing, so the data can be an integrating point for the combined application of both.

Intelligent processing provides some data acquisition methods, data and information analysis modes, supervises thestate of the renewable resources, such as position regulation, so it can be used for tracking and traceability of renewable resources, huge amounts of data can be collected for subsequent comprehensive analysis, and it can also provide decision support andsome necessary data for life cycle assessment (mainly phase LCI). However, intelligent processing mainly provides technical support, but cannot provide environmental impact assessment.Life cycle analysis focuses on the environmental impact assessment of the entire process. Some of the data required by the lifecycle analysis can be provided by intelligent processing, while other data will be collected in other ways, and finally, an environmental impact assessment report will be given to provide decision makers with recommendations for optimizing environmental impacts.

Technological progress and people's growing demand requires life cycle analysis to develop towards real-time and field life cycle analysis, demands the real-time field and assessment of the environmental impact of items, which is essentially equivalent to shorten the time step of life cycle analysis. Therefore, it can more accurately evaluate the environmental impact of the items than the original analysis methods and monitoring methods. The development of intelligent processing provides a good monitoring means for this requirement of lifecycle analysis. Specifically, the developed intelligent processing technology provides more accurate data in time dimension for the lifecycle analysis method, so that researchers can achieve the lifecycle analysis meeting the accuracy requirementsin a relatively small time step.

\section{Conclusion}

As for the current development level of science and technology, intelligent processing can provide some data support for life cycle analysis.At present, the combined application of intelligent processing and life cycle analysis is almost blank. The fundamental reason is that the development level of the current intelligent processing technology cannot meet the needs of short time step life cycle analysis. Meanwhile, intelligent processing has not been fully deployed in the global scope, and the environment of "intelligent processing" is not available for the time being.This makes it difficult to realize the combined application of intelligent processing and lifecycle analysis at scale.Technology is developing at an accelerated pace. It is believed that in the near future, intelligent processing and life cycle analysis will be closely combined, which will play an irreplaceable role not only in the field of renewable resources management but also in many aspects of social production and human life.

\section{Reference}

[1] Wang Beibei, Zhao Shengnan, Liu XiaoCong, et al. Analysis and Thinking on Key Technologies of smart power consumption for renewable energy consumption [J]. Power grid technology, 2016, 40 (12): 3894-3903

[2] Wang Shoubing. Life cycle ecological assessment of complex industrial products in China -- Method 
and case study [D]. Fudan University

[3] Ye min, Wan houfen. Product life cycle analysis based on circular economy [C] / / 2006 annual meeting of China Marketing Society and the fourth national member congress. 0

[4] Yu Jing, Wang Hui. Application of big data and its intelligent processing technology in the Internet of things industry [J]. Silicon Valley, 2013, 000 (017): 1-1

[5] Cao Yingying. Construction of Intelligent Community guided by information intelligent processing technology [J]. Computer technology and development, 2015, 000 (001): 207-211

[6] Chen Yihang, Yu ZHENGJING. Analysis of dynamic characteristics of product life cycle [J]. Chemical industry, 2002, 49 (4): 80-91

[7] Wu Shufang. Research on cost-benefit optimization of reverse logistics based on environmental accounting [D]. Harbin University of Commerce, 2012

[8] Han Lida. Penetration mechanism of high and new technologies and advanced applicable technologies into traditional industries based on product life cycle analysis [J]. Scientific management research, 2002, 20 (004): 27-31 